\title{
Machine Learning for Mental Health in Social Media: Bibliometric Study
}

Jina Kim, BSc, MSc; Daeun Lee, BA; Eunil Park, BSc, MSc, PhD

Department of Applied Artificial Intelligence, Sungkyunkwan University, Seoul, Republic of Korea

\section{Corresponding Author:}

Eunil Park, BSc, MSc, PhD

Department of Applied Artificial Intelligence

Sungkyunkwan University

312 International Hall

Sungkyunkwan-ro 25-2

Seoul, 03063

Republic of Korea

Phone: 8227401864

Fax: 8227401856

Email: eunilpark@skku.edu

\section{Abstract}

Background: Social media platforms provide an easily accessible and time-saving communication approach for individuals with mental disorders compared to face-to-face meetings with medical providers. Recently, machine learning (ML)-based mental health exploration using large-scale social media data has attracted significant attention.

Objective: We aimed to provide a bibliometric analysis and discussion on research trends of ML for mental health in social media.

Methods: Publications addressing social media and ML in the field of mental health were retrieved from the Scopus and Web of Science databases. We analyzed the publication distribution to measure productivity on sources, countries, institutions, authors, and research subjects, and visualized the trends in this field using a keyword co-occurrence network. The research methodologies of previous studies with high citations are also thoroughly described.

Results: We obtained a total of 565 relevant papers published from 2015 to 2020. In the last 5 years, the number of publications has demonstrated continuous growth with Lecture Notes in Computer Science and Journal of Medical Internet Research as the two most productive sources based on Scopus and Web of Science records. In addition, notable methodological approaches with data resources presented in high-ranking publications were investigated.

Conclusions: The results of this study highlight continuous growth in this research area. Moreover, we retrieved three main discussion points from a comprehensive overview of highly cited publications that provide new in-depth directions for both researchers and practitioners.

(J Med Internet Res 2021;23(3):e24870) doi: 10.2196/24870

\section{KEYWORDS}

bibliometric analysis; machine learning; mental health; social media

\section{Introduction}

\section{Background}

Artificial intelligence (AI) has permeated various daily sectors that are directly related to our lives [1,2]. With this trend, AI for health, which refers to applying AI to real-world health care, has become one of the most important social issues at present $[3,4]$. With privacy and security as the bedrock of AI-based health care, there have been many attempts to employ AI and its applications in health care services [5,6]. As a representative example, Rizwan Malik, a radiologist in the United Kingdom, adopted a unique AI-based chest X-ray system to reduce patient waiting time in the COVID-19 pandemic scenario [4]. Furthermore, Microsoft [2] has invested approximately US \$20 million to aid the collaboration teams of health care professionals and data science/AI experts in COVID-19-related research. 
Extensive efforts have been put forward to employ AI technologies in health care services in addressing issues related to physical health, involving several medical centers, researchers, and organizations, as well as for mental health as a rapidly growing social issues. Although mental health is a pervasive and comprehensive issue, its detection and exposure are challenging. The World Health Organization estimates that approximately 1 billion people worldwide have mental disorders [7]. Moreover, 264 million people have been globally affected by depression, a common mental disorder [8]. However, more than $75 \%$ of people in underdeveloped countries (ie, low-income countries) suffering from mental disorders do not receive any treatments [7]. Several scholars have also revealed that individuals who suffer from mental disorders tend to prefer sharing their personal information and seeking assistance to reduce their concerns through online channels rather than with medical providers such as counselors or therapists [9-11].

Considering this tendency, social media represents a supportive tool for these individuals [11], where users are allowed to generate content, share information, and communicate [12]. Many researchers have attempted to explore the large-scale user-generated content in social media by means of machine learning (ML), which is a robust data-engineering technique, to analyze hidden information and knowledge on mental health. Therefore, we provide a theoretical background of related studies in the following subsections.

\section{Related Review Papers}

Higgins et al [13] define a systematic review as

a study that is composed of a search for scientific publications related to various topics in accordance with systematic guidelines including the search queries, the scientific databases, and the assessment criteria.

With this concept, several prior mental health studies have investigated how to utilize ML in social media datasets. For instance, Seabrook et al [14] examined a systematic approach to provide an overview of prior research that focused on depression and anxiety in social media contexts between 2005 and 2016 with 8 identified databases. To objectively evaluate and summarize the literature, each case was evaluated by three unique dimensions: how to include psychological/cognitive measures, how to use external measurements for mental health criteria, and how to collect user activities in social media. Subsequently, 70 cases were selected, examined, and reviewed for both the implications and future directions regarding the application of ML to mental health in social media.

In addition to a systematic review approach, a scoping review may be performed, which is defined as "a type of research synthesis that aims to map the literature on a particular topic or research area and provide an opportunity to identify key concepts" [15]. This implies that a scoping review provides a bird's eye view of key concepts in specific research areas, main sources, findings, and implications. For instance, Shatte et al [16] adopted a scoping review approach including 300 papers that focused on ML and big data applications in mental health, and concluded that the majority of these papers considered depression, schizophrenia, and Alzheimer disease as their main mental illnesses. Moreover, $89 \%$ of the papers analyzed utilized supervised learning approaches such as support vector machine (SVM), naïve Bayes, or decision trees to examine their selected illness.

Chancellor et al [17] also performed a thematic discourse analysis on 55 scientific papers with the goal of predicting mental health status in social media, and demonstrated that interdisciplinary researchers have different perspectives toward users' datasets; these perspectives were classified as "human-centered machine learning" (HCML). Based on these findings and the concept of HCML, Chancellor and De Choudhury [18] subsequently categorized a total of 75 papers with five discourses: disorder/patient, social media, scientific, data/ML, and person. Based on this categorization, a total of 75 cases in which mental health status was assessed using social media datasets within 41 conference/journal papers published from 2013 to 2018 retrieved through academic databases (eg, ACM Digital Library and Google Scholar) were reviewed with respect to data annotation methods, data collection/quality management, preprocessing procedures, feature selections, model selection, and verification.

As presented in numerous prior studies, ML and mental health in social media have gained exponential attention in both practical and academic fields. Thus, we aimed to perform a bibliometric analysis to provide an overview and recent trends of this field.

\section{Related Bibliometric Analyses}

Bibliometric analysis is an extensive and widely used approach "to shed light on the processes of written communication and of the nature and course of development of a discipline" [19]. A bibliometric analysis thus allows researchers to understand the trends of specific research areas with several primary publications, including collaboration relations [20,21], core research themes [20], and scientific techniques [22].

Several scholars have performed bibliometric analyses on $\mathrm{AI} / \mathrm{ML}$ in health care areas, including public and mental health, as well as in areas of specific mental illnesses. For example, dos Santos et al [22] performed a bibliometric analysis of data mining and ML techniques applied to public health issues based on papers published between 2009 and 2018 retrieved from three academic databases: Web of Science (WoS), Scopus, and ScienceDirect.

In the case of depression specifically, Tran et al [23] used a bibliometric approach to examine AI applications presented in publications indexed in WoS, evaluated the productivity of AI research through statistical analyses, and performed an exploratory factor analysis on the contexts of paper abstracts to present the most relevant and popular research issues. Moreover, Wang et al [24] performed a bibliometric analysis of natural language processing in various medical research areas including papers retrieved from PubMed data engines published from 1999 to 2018.

With respect to social media, several bibliometric analyses have closely evaluated relevant publications and their effects on society. As a representative example, Chen et al [20] adopted 
both quantitative and statistical approaches with the WoS database to detect specific events in social media within the period of 2009-2017, investigated the number of publications and degree of collaboration, and further used clustering analysis to identify the main research themes. Another bibliometric study conducted by Sa'ed et al [21] focused on social media in psychology over 12 years based on records retrieved from WoS, and identified bibliometric indicators, including international collaboration/research networks.

Based on this background, the use of ML in medical fields and social media has been extensively explored using bibliometric approaches with notable implications and future directions. Therefore, this is an appropriate time to provide more detailed observations on ML with respect to the relation of specific medical areas with social media. Specifically, we examined the trends of research using ML for mental health in social media by employing (1) a bibliometric analysis to determine the publication distributions on sources (journals or conferences), authors, institutions, countries, research subjects, and author keywords; and (2) a trend review analysis to determine the distributions of citation numbers, along with a comprehensive review of highly cited publications.

With these approaches, we aimed to identify overall research trends of this area in a quantitative manner, and to qualitatively identify the key methodologies used on diverse social media platforms. These findings can shed light on the recent trends in the field and highlight more detailed directions of future research areas.

\section{Methods}

\section{Data Collection}

We collected papers from two citation databases, Scopus and WoS. Scopus is one of the largest citation repositories that covers scientific journals, conference proceedings, and books. WoS stores high-quality publications evaluated by three main indices: Science Citation Index Expanded (SCIE), Social Sciences Citation Index (SSCI), or Art \& Humanities Citation Index (A\&HCI).

Relevant publications were obtained when the terms included in the search query appeared in the title, abstract, or keywords. We defined the search query of each topic based on prior research on ML, social media [17], and mental health [25]. We excluded papers that were not written in English or were categorized as other document types (Figure 1). As a result, a total of 565 papers published from 2016 to 2020 were obtained on July 21, 2020. To cover rapidly changing trends in ML areas, we also considered the year 2020, which is still open for new issues. The complete list of included publications is provided in Multimedia Appendix 1.

Figure 1. Representative data collection procedure.

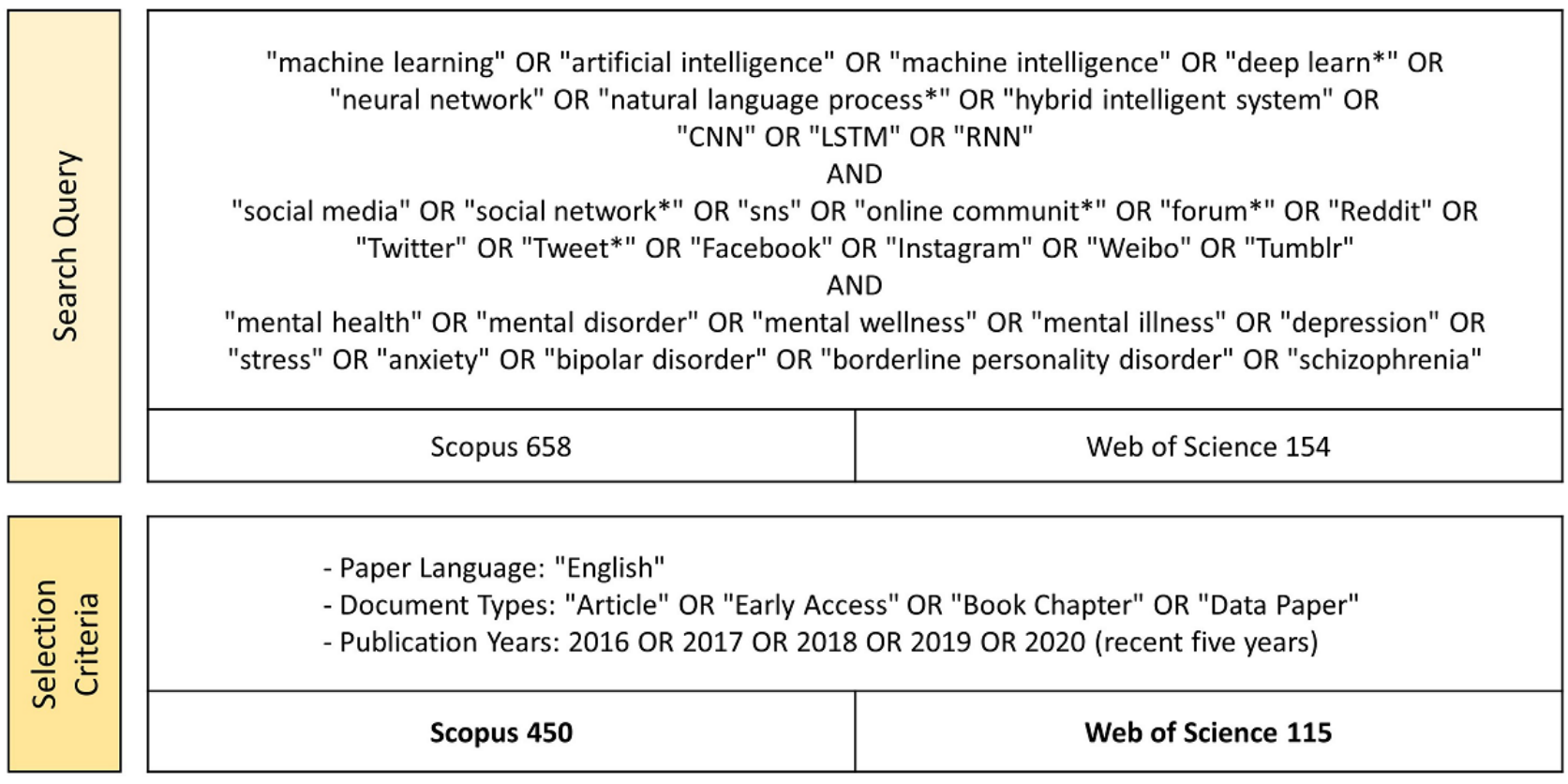

*Retrieved Date: July 21, 2020

\section{Analysis Methodologies}

A bibliometric analysis includes the distribution exploration of publication and research subject, as well as citation quantities. Both the Python programming language and Microsoft Excel were employed to perform statistical analyses of the retrieved papers. We first analyzed the publication distributions of papers with several categories (eg, sources, countries, institutions, authors, and research subjects). We also performed a network analysis of frequently used keywords. Moreover, to identify the research trends in this area, we performed trend review analyses with highly cited papers covering the following topics: (i) ML techniques, (ii) specific mental illnesses, and (iii) social media. 


\section{Results}

\section{Publication Distribution Analysis}

\section{Overall Publication Trend}

The continuous growth of publications from 2016 to 2020 (until
July 2020) is illustrated in Table 1. In 2016, two papers were retrieved from WoS and 33 papers were retrieved from Scopus. The publication count demonstrates rapid growth in 2019 with 43 publications retrieved from WoS and 166 publications retrieved from Scopus. Considering the retrieved date (July 2020), we expect that more papers would be retrieved in the remainder of 2020 up to the present.

Table 1. Number of publications per year.

\begin{tabular}{lll}
\hline Year & Publication count, $\mathrm{n}(\%)$ & \\
& Scopus $(\mathrm{N}=450)$ & $2(1.7)$ \\
2016 & $33(7.3)$ & $16(13.9)$ \\
2017 & $68(15.1)$ & $21(18.3)$ \\
2018 & $88(19.6)$ & $43(37.4)$ \\
2019 & $166(36.9)$ & $33(28.7)$ \\
\hline
\end{tabular}

\section{Productive Publication Source}

We considered several document types, including not only journal articles but also conference proceedings and book chapters. Tables 2 and 3 present the publication sources with high counts in Scopus and WoS, respectively. Lecture Notes in Computer Science was the most productive publication source in Scopus, followed by CEUR Workshop Proceedings, Neural Computing and Applications, and Journal of Medical Internet Research with more than 20 publication counts each. Journal of Medical Internet Research was selected as the most productive publication source in WoS with 15 publication counts, followed by IEEE Access.

Table 2. Top publication sources in Scopus ( $\mathrm{N}=450)$.

\begin{tabular}{lll}
\hline Rank & Source & Publication count, $\mathrm{n}(\%)$ \\
\hline 1 & Lecture Notes in Computer Science & $35(7.8)$ \\
2 & CEUR Workshop Proceedings & $25(5.6)$ \\
3 & Neural Computing and Applications & $22(4.9)$ \\
4 & Journal of Medical Internet Research & $16(3.6)$ \\
5 & Advances in Intelligent Systems and Computing & $13(2.9)$ \\
6 & ACM International Conference Proceeding Series & $9(2.0)$ \\
6 & International Journal of Innovative Technology and Exploring Engineering & $9(2.0)$ \\
8 & IEEE Access & $7(1.6)$ \\
9 & Communications in Computer and Information Science & $5(1.1)$ \\
9 & Frontiers in Psychiatry & $5(1.1)$ \\
9 & International Journal of Environmental Research and Public Health & $5(1.1)$
\end{tabular}


Table 3. Top publication sources in Web of Science $(\mathrm{N}=115)$.

\begin{tabular}{lll}
\hline Rank & Source & Publication count, $\mathrm{n}(\%)$ \\
\hline 1 & Journal of Medical Internet Research & $15(13.0)$ \\
2 & IEEE Access & $7(6.1)$ \\
3 & BMJ Open & $3(2.6)$ \\
3 & Computers in Human Behavior & $3(2.6)$ \\
3 & Frontiers in Psychology & $3(2.6)$ \\
3 & IEEE Transactions on Knowledge and Data Engineering & $3(2.6)$ \\
3 & International Journal of Environmental Research and Public Health & $3(2.6)$ \\
8 & BMC Medical Informatics and Decision Making & $2(1.7)$ \\
8 & Cyberpsychology Behavior and Social Networking & $2(1.7)$ \\
8 & Journal of Information Science & $2(1.7)$ \\
8 & Journal of Intelligent information Systems & $2(1.7)$ \\
8 & Multimedia Tools and Applications & $2(1.7)$ \\
8 & NPJ Schizophrenia & $2(1.7)$ \\
8 & Scientific Reports & $2(1.7)$ \\
8 & Social Science Computer Review & $2(1.7)$ \\
8 & Translational Behavioral Medicine & $2(1.7)$
\end{tabular}

\section{Predominant Countries}

More than 30 countries were identified as the predominant nations performing research in this field in Scopus $(n=59)$ and
WoS $(n=39)$. Table 4 illustrates the top productive countries based on the number of publications. The United States was the most productive nation in both databases, followed by China and India. 
Table 4. Top productive countries.

\begin{tabular}{|c|c|c|}
\hline Rank & Country & Publication count, $\mathrm{n}(\%)$ \\
\hline \multicolumn{3}{|c|}{ Scopus $(\mathbf{N}=\mathbf{4 5 0})$} \\
\hline 1 & United States & $146(32.4)$ \\
\hline 2 & India & $66(14.7)$ \\
\hline 3 & China & $63(14.0)$ \\
\hline 4 & United Kingdom & $34(7.6)$ \\
\hline 5 & Canada & $22(4.9)$ \\
\hline 6 & Spain & $18(4.0)$ \\
\hline 7 & Australia & $17(3.8)$ \\
\hline 8 & Germany & $16(3.6)$ \\
\hline 9 & Taiwan & $14(3.1)$ \\
\hline 10 & France & $13(2.9)$ \\
\hline 10 & Netherlands & $13(2.9)$ \\
\hline \multicolumn{3}{|c|}{ Web of Science $(\mathrm{N}=115)$} \\
\hline 1 & United States & $52(45.2)$ \\
\hline 2 & China & $25(21.7)$ \\
\hline 3 & United Kingdom & $12(10.4)$ \\
\hline 4 & Australia & $11(9.6)$ \\
\hline 5 & Spain & $6(5.2)$ \\
\hline 6 & Canada & $5(4.4)$ \\
\hline 6 & India & $5(4.4)$ \\
\hline 6 & Saudi Arabia & $5(4.4)$ \\
\hline 7 & South Korea & $4(3.5)$ \\
\hline 7 & Taiwan & $4(3.5)$ \\
\hline
\end{tabular}

\section{Productive Institutions}

There were 391 different institutions associated with the 565 publications. The top-ranked institutions are presented in Table
5. Harvard University in the United States emerged as the most productive institution in WoS (13 publications), whereas Tsinghua University in China was selected as the most productive organization in Scopus (21 publications). 
Table 5. Top productive institutions.

\begin{tabular}{|c|c|c|}
\hline \multirow[t]{2}{*}{ Institution } & \multicolumn{2}{|c|}{ Publication count, $\mathrm{n}(\%)$} \\
\hline & Scopus $(\mathrm{N}=450)$ & Web of Science $(\mathrm{N}=115)$ \\
\hline Tsinghua University & $21(4.7)$ & $3(2.6)$ \\
\hline Georgia Institute of Technology & $12(2.7)$ & $4(3.5)$ \\
\hline Harvard University & $14(3.1)$ & $13(11.3)$ \\
\hline University of Pennsylvania & $13(2.9)$ & $5(4.4)$ \\
\hline Chinese Academy of Sciences & $14(3.1)$ & $6(5.2)$ \\
\hline Johns Hopkins University & $6(1.3)$ & $3(2.6)$ \\
\hline King's College London & $6(1.3)$ & $-^{\mathrm{a}}$ \\
\hline University of Toronto & $6(1.3)$ & $3(2.6)$ \\
\hline National Tsing Hua University & $6(1.3)$ & $3(2.6)$ \\
\hline Northwestern University & $5(1.1)$ & $1(0.9)$ \\
\hline Centre National de la Recherche Scientifique & $5(1.1)$ & - \\
\hline Vrije Universiteit Amsterdam & $5(1.1)$ & - \\
\hline The University of Arizona & $5(1.1)$ & - \\
\hline National University of Singapore & $5(1.1)$ & $1(0.9)$ \\
\hline Deakin University & $5(1.1)$ & $3(2.6)$ \\
\hline University of New South Wales & $5(1.1)$ & $1(0.9)$ \\
\hline Ministry of Education China & $4(0.9)$ & - \\
\hline Delhi Technological University & $4(0.9)$ & - \\
\hline Cornell University & $4(0.9)$ & $1(0.9)$ \\
\hline Radboud University Nijmegen & $4(0.9)$ & - \\
\hline Russian Academy of Sciences & $4(0.9)$ & - \\
\hline Microsoft Research & $4(0.9)$ & - \\
\hline The University of Utah & $4(0.9)$ & $1(0.9)$ \\
\hline Universidad Autónoma de Madrid & $4(0.9)$ & - \\
\hline University of Rochester & $4(0.9)$ & - \\
\hline University of Chinese Academy of Sciences & $4(0.9)$ & $3(2.6)$ \\
\hline Université du Québec à Montréal & $4(0.9)$ & $2(1.7)$ \\
\hline King Faisal University & $4(0.9)$ & $2(1.7)$ \\
\hline University of Texas System & $4(0.9)$ & - \\
\hline Asia University Taiwan & $4(0.9)$ & - \\
\hline
\end{tabular}

a__no related records.

\section{Predominant Authors}

The top 20 researchers contributing to the field are listed in Table 6 based on their number of publications. Twelve researchers are affiliated to US-based organizations and five belong to Chinese institutions. The institutions of productive

authors include not only several academic institutions but also some well-known hospitals such as Zucker Hillside Hospital. The most productive researcher was Professor Munmun De Choudhury, affiliated with Georgia Institute of Technology (15 publications), followed by Professor Sharath Chandra Guntuku from the University of Pennsylvania. 
Table 6. Top 20 productive authors.

\begin{tabular}{|c|c|c|c|c|}
\hline \multirow[t]{2}{*}{ Author } & \multirow[t]{2}{*}{ Institution } & \multirow[t]{2}{*}{ Country } & \multicolumn{2}{|c|}{ Publication count, n (\%) } \\
\hline & & & $\begin{array}{l}\text { Scopus } \\
(\mathrm{N}=450)\end{array}$ & $\begin{array}{l}\text { Web of Science } \\
(\mathrm{N}=115)\end{array}$ \\
\hline M De Choudhury & Georgia Institute of Technology & United States & $11(2.4)$ & $4(3.5)$ \\
\hline SC Guntuku & University of Pennsylvania & United States & $5(1.1)$ & $4(3.5)$ \\
\hline HF Ahmad & King Faisal University & Saudi Arabia & $4(0.9)$ & $3(2.6)$ \\
\hline SK Ernala & Georgia Institute of Technology & United States & $4(0.9)$ & $3(2.6)$ \\
\hline LH Ungar & University of Pennsylvania & United States & $4(0.9)$ & $3(2.6)$ \\
\hline T Nguyen & University of Pennsylvania & United States & $5(1.1)$ & $2(1.7)$ \\
\hline S Venkatesh & University of Maryland & United States & $5(1.1)$ & $2(1.7)$ \\
\hline ML Birnbaum & Zucker Hillside Hospital & United States & $4(0.9)$ & $2(1.7)$ \\
\hline M Conway & University of Utah & United States & $4(0.9)$ & $2(1.7)$ \\
\hline L Feng & Tsinghua University & China & $4(0.9)$ & $2(1.7)$ \\
\hline D Phung & Deakin University & Australia & $4(0.9)$ & $2(1.7)$ \\
\hline J Jia & Tsinghua University & China & $6(1.3)$ & $-^{\mathrm{a}}$ \\
\hline T Zhu & Chinese Academy of Sciences & China & $6(1.3)$ & - \\
\hline RM Merchant & University of Pennsylvania & United States & $2(0.4)$ & $3(2.6)$ \\
\hline H Christensen & University of New South Wales & Australia & $3(0.7)$ & $2(1.7)$ \\
\hline JM Kane & Zucker Hillside Hospital & United States & $3(0.7)$ & $2(1.7)$ \\
\hline Q Li & Tsinghua University & China & $3(0.7)$ & $2(1.7)$ \\
\hline AF Rizvi & Zucker Hillside Hospital & United States & $3(0.7)$ & $2(1.7)$ \\
\hline CY Shen & National Tsing Hua University & China & $3(0.7)$ & $2(1.7)$ \\
\hline L Ungar & University of Pennsylvania & United States & $3(0.7)$ & $2(1.7)$ \\
\hline
\end{tabular}

a_: no related records.

\section{Productive Research Subjects}

The top 10 research subjects of each citation database are given in Figure 2. Among them, computer science was the most pivotal research subject in both databases $(46,40 \%$ in WoS; $292,65 \%$

Figure 2. Publication count of top 10 research subjects.

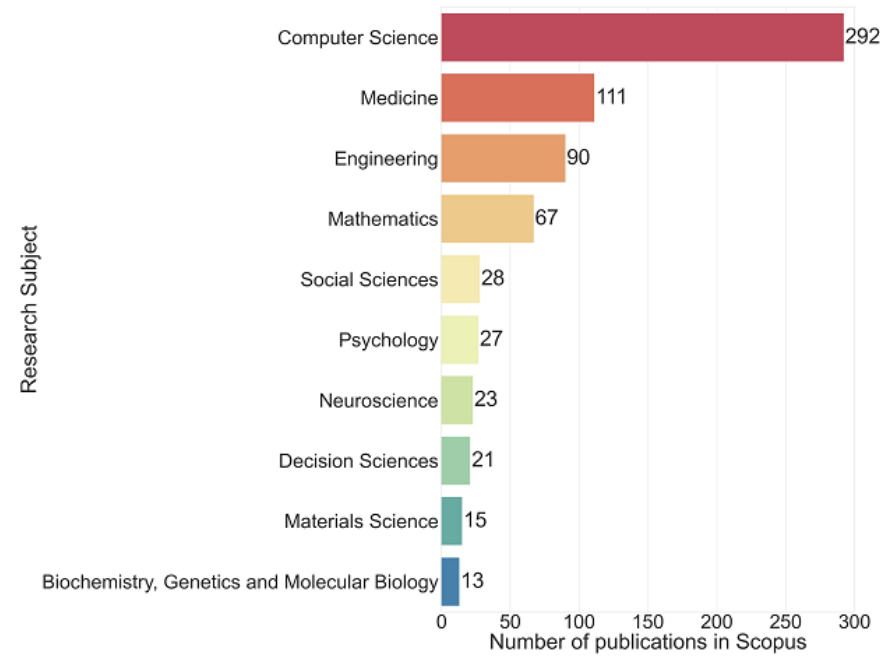

in Scopus). In Scopus, medicine (111, 25\%), and engineering and mathematics $(90,20 \%)$ accounted for more than $10 \%$ of the total publications. In WoS, medical informatics $(24,21 \%)$, health care sciences services, engineering, and psychology constituted more than $10 \%$ of total publications. 


\section{Author Keyword Co-occurrence}

Along with research subjects, we investigated the keywords derived by the authors, which represent the main research topics of the publications [26]. The keyword co-occurrence is visualized using a network graph (Figure 3), which is a well-known bibliometric methodology, in which each node is a keyword, while an edge between two nodes indicates the co-occurrence of two words. After building a network graph, we excluded edges with less than 3 co-occurrences.

Figure 3. Keyword co-occurrence network graph; the color map on the right side represents the degree centrality.

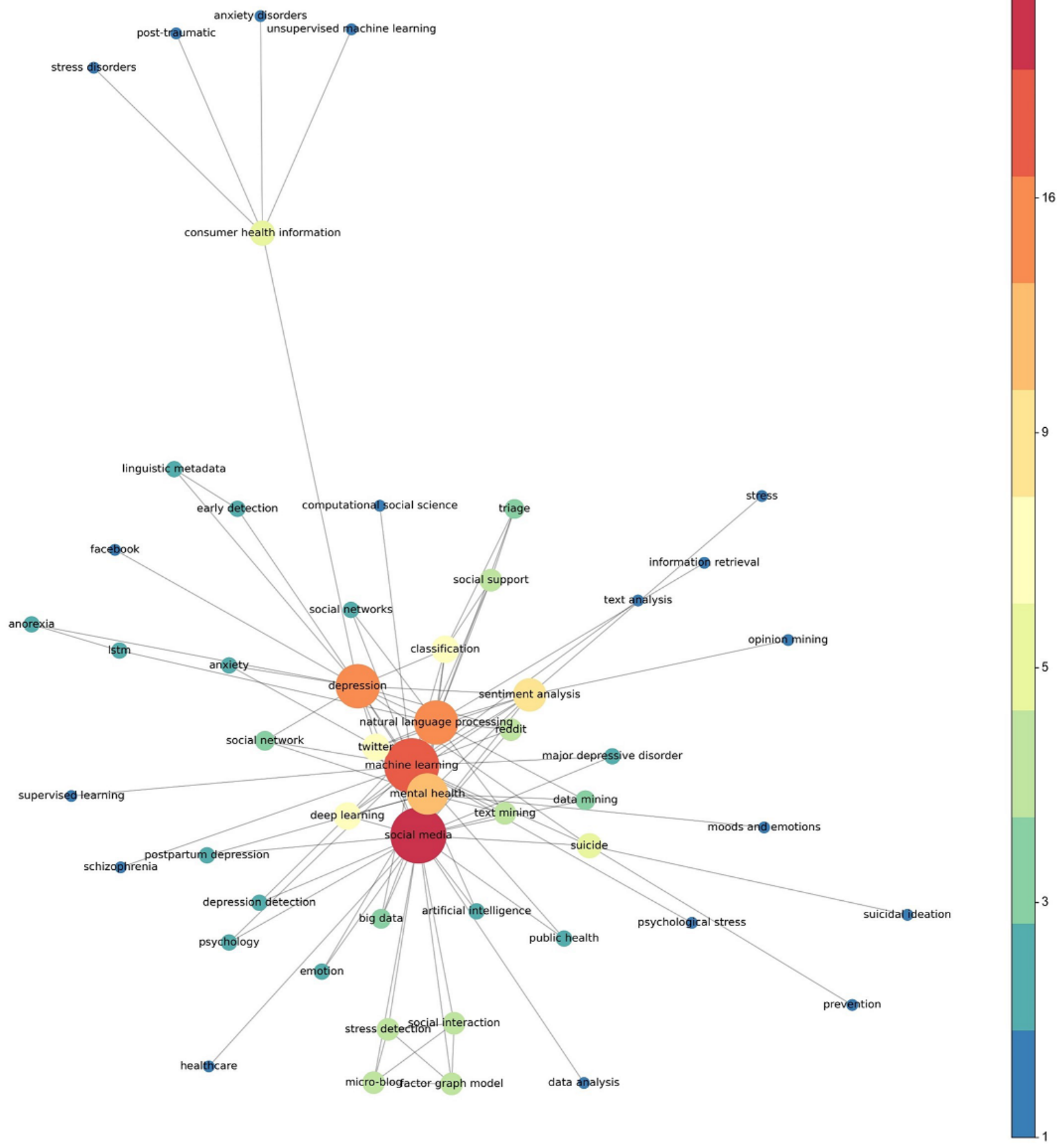

Note that the keywords with a high frequency reflect the scope of the research area, which includes social media, ML, natural language processing, and mental health. In particular, in the case of mental health-related words, depression was the most frequently presented keyword, followed by suicide, consumer health information, social support, and stress detection. The main research methodologies of papers included natural language processing, sentiment analysis, classification, text mining, and stress detection in the ML field. Twitter and Reddit were identified as the most widely investigated social media platforms in this area.

As there are several approaches of text analysis using natural language processing techniques in this field, we believe that future studies may provide hybrid approaches using both textualand visual-based data collected from several types of social 
media data. In addition, validating ML models trained on mental health-related social media data in clinical settings needs to be further investigated.

\section{Overview of Highly Cited Publications}

\section{Publication Citation Quantities}

The annual number of citations is presented in Table 7. Along with publication distributions, the annual number of citations has been consistently increasing. Up to July 21, 2020, more than 900 and 400 annual citations were recorded in Scopus and WoS, respectively.

Table 7. Number of citations per year.

\begin{tabular}{lll}
\hline Year & Citation count, $\mathrm{n}$ & \\
& Scopus & Web of Science \\
\hline 2016 & 14 & 1 \\
2017 & 112 & 32 \\
2018 & 349 & 120 \\
2019 & 1000 & 341 \\
2020 & 938 & 420 \\
\hline
\end{tabular}

\section{Comprehensive Analysis of Highly Cited Papers}

We evaluated the overall academic output through bibliometric analysis. Due to the lack of observations in the content of the publications, as mentioned in previous studies [20,27], we extensively observed and reviewed the top five most highly cited papers per year to identify the comprehensive research methodologies in the field (Table 8). After excluding 7 duplicated papers, 39 papers were selected. Subsequently, a two-round filtering procedure was performed to determine whether a specific paper meets the following criteria: (i) addressing specific mental illness, (ii) using an ML technique, and (iii) utilizing datasets of social media. There were 15 papers that met these criteria. Subsequently, three experts in ML, medical services, and computer science, respectively, participated in the second-round filtering procedure. Following this, 10 papers that satisfied these criteria were selected.

Table 8. Overview of the top 5 most cited papers by year.

\begin{tabular}{lllll}
\hline Year & $\begin{array}{l}\text { Top-cited papers }(\mathrm{N}=39), \mathrm{n} \\
\end{array}$ & Article & Conference paper & Article \\
\hline 2016 & 5 & 2 & 2 & 1 \\
2017 & 6 & 0 & 3 & 0 \\
2018 & 9 & 0 & 2 & 0 \\
2019 & 8 & 2 & 1 & 0 \\
2020 & 6 & 1 & 1 & 0
\end{tabular}

An overview and the research methodologies of the 10 highly cited publications are listed in Table 9, which are categorized according to the data source: Twitter ( $n=4$ publications),
Instagram $(n=3)$, Facebook $(n=3)$, Reddit $(n=3)$, Weibo $(n=2)$, and other online community $(\mathrm{n}=1)$. 
Table 9. Summary of research methodologies employed in highly cited publications.

\begin{tabular}{|c|c|c|c|c|c|c|c|c|}
\hline \multirow[t]{2}{*}{ Data Source } & \multirow[t]{2}{*}{ Reference } & \multirow{2}{*}{$\begin{array}{l}\text { Mental } \\
\text { Health }\end{array}$} & \multirow{2}{*}{$\begin{array}{l}\text { Data Descrip- } \\
\text { tion }\end{array}$} & \multicolumn{5}{|l|}{ Machine learning } \\
\hline & & & & Model & Feature & Output & Annotation & Results \\
\hline Twitter & $\begin{array}{l}\text { Budenz et al } \\
{[28]}\end{array}$ & $\begin{array}{l}\text { Mental ill- } \\
\text { ness, bipolar } \\
\text { disorder }\end{array}$ & $\begin{array}{l}1,270,902 \\
\text { tweets includ- } \\
\text { ing bipolar or } \\
\text { mental health- } \\
\text { related words }\end{array}$ & $\begin{array}{l}\text { Logistic regres- } \\
\text { sion }\end{array}$ & $\begin{array}{l}\text { Term frequen- } \\
\text { cy-inverse docu- } \\
\text { ment frequency }\end{array}$ & $\begin{array}{l}\text { Related to } \\
\text { mental ill- } \\
\text { ness or bipo- } \\
\text { lar disorder }\end{array}$ & $\begin{array}{l}\text { Manually anno- } \\
\text { tated } 2047 \\
\text { tweets with top- } \\
\text { ic, stigma, and } \\
\text { social support } \\
\text { messaging }\end{array}$ & $\begin{array}{l}10 \text {-fold cross } \\
\text { validation } \\
\left(\mathrm{AUC}^{\mathrm{a}}=0.83\right)\end{array}$ \\
\hline Twitter & Du et al [29] & Suicide & $\begin{array}{l}1,962,766 \\
\text { tweets includ- } \\
\text { ing } 21 \text { suicide- } \\
\text { related key- } \\
\text { words/phrases }\end{array}$ & $\begin{array}{l}\mathrm{CNN}^{\mathrm{b}}, \mathrm{SVM}^{\mathrm{c}}, \\
\text { extra trees, ran- } \\
\text { dom forest, lo- } \\
\text { gistic regres- } \\
\text { sion, Bi-LSTM }\end{array}$ & $\begin{array}{l}\text { One-hot-vector } \\
\text { mapped to pre- } \\
\text { trained GloVe } \\
\text { Twitter embed- } \\
\text { ding }\end{array}$ & $\begin{array}{l}\text { Related to } \\
\text { suicide }\end{array}$ & $\begin{array}{l}\text { Manually anno- } \\
\text { tated } 3263 \\
\text { tweets and } \\
\text { trained classifi- } \\
\text { er to select } \\
3000 \text { additional } \\
\text { suicide-related } \\
\text { tweets }\end{array}$ & $\begin{array}{l}\text { Accuracy } 0.74 \text {, } \\
\text { recall } 0.96, \text { pre- } \\
\text { cision } 0.78, \mathrm{~F} 1 \\
0.83\end{array}$ \\
\hline $\begin{array}{l}\text { Facebook, } \\
\text { Twitter }\end{array}$ & $\begin{array}{l}\text { Guntuku et } \\
\text { al [30] }\end{array}$ & $\begin{array}{l}\text { Psychologi- } \\
\text { cal stress }\end{array}$ & $\begin{array}{l}601 \text { users' } \\
\text { Facebook and } \\
\text { Twitter posts }\end{array}$ & $\begin{array}{l}\text { Linear regres- } \\
\text { sion with sever- } \\
\text { al regularization } \\
\text { methods (eg, } \\
\text { ridge, elastic- } \\
\text { net, LASSO }{ }^{\mathrm{e}} \\
\text { and L2 penal- } \\
\text { ized SVMs) }\end{array}$ & $\begin{array}{l}\text { LIWC } \text {, latent } \\
\text { Dirichlet alloca- } \\
\text { tion topic model- } \\
\text { ing, stress lexi- } \\
\text { con, user en- } \\
\text { gagement }\end{array}$ & Stress & $\begin{array}{l}\text { Qualtrics sur- } \\
\text { vey; fill out the } \\
\text { demographic } \\
\text { questions and } \\
\text { Cohen 10-item } \\
\text { Stress Scale }\end{array}$ & $\begin{array}{l}\text { 5-fold cross- } \\
\text { validation } \\
\text { (Pearson } \\
r=0.24 \text { ); trained } \\
\text { on Facebook } \\
\text { and Twitter, } \\
\text { tested on Twit- } \\
\text { ter }\end{array}$ \\
\hline $\begin{array}{l}\text { Facebook, } \\
\text { Instagram }\end{array}$ & $\begin{array}{l}\text { Shuai et al } \\
\text { [31] }\end{array}$ & $\begin{array}{l}\text { Social net- } \\
\text { work mental } \\
\text { disorders } \\
\text { (eg, cyber- } \\
\text { relationship } \\
\text { addiction, in- } \\
\text { formation } \\
\text { overload, } \\
\text { and net com- } \\
\text { pulsion) }\end{array}$ & $\begin{array}{l}3126 \text { users' In- } \\
\text { stagram and } \\
\text { Facebook data }\end{array}$ & $\begin{array}{l}\text { Decision tree } \\
\text { learning, SVM, } \\
\text { logistic regres- } \\
\text { sion, DTSVM } \\
\text { SNMDD }^{\mathrm{h}} \\
\text { (newly pro- } \\
\text { posed model; } \\
\text { tensor tech- } \\
\text { nique for deriv- } \\
\text { ing latent fea- } \\
\text { tures) }\end{array}$ & $\begin{array}{l}\text { Social interac- } \\
\text { tion, personal } \\
\text { profile, duration }\end{array}$ & $\begin{array}{l}\text { Social net- } \\
\text { work mental } \\
\text { disorders }\end{array}$ & $\begin{array}{l}\text { MTurk survey } \\
\text { - fill out the } \\
\text { standard social } \\
\text { network mental } \\
\text { disorder ques- } \\
\text { tionnaires; pro- } \\
\text { fessional psychi- } \\
\text { atrists labeled } \\
\text { users having a } \\
\text { social network } \\
\text { mental disorder }\end{array}$ & $\begin{array}{l}\text { 5-fold cross val- } \\
\text { idation (accura- } \\
\text { cy0.78 for Insta- } \\
\text { gram and } 0.83 \\
\text { for Facebook) }\end{array}$ \\
\hline Instagram & $\begin{array}{l}\text { Reece and } \\
\text { Danforth } \\
{[32]}\end{array}$ & Depression & $\begin{array}{l}43,950 \text { users' } \\
\text { Instagram im- } \\
\text { ages }\end{array}$ & $\begin{array}{l}\text { Random forest } \\
\text { classifier }\end{array}$ & $\begin{array}{l}\text { Number of } \\
\text { comments and } \\
\text { likes, number of } \\
\text { faces in photo- } \\
\text { graph, } 3 \text { color } \\
\text { properties (hue, } \\
\text { saturation, val- } \\
\text { ue) }\end{array}$ & Depression & $\begin{array}{l}\text { MTurk survey; } \\
\text { Center for Epi- } \\
\text { demiologic } \\
\text { Studies Depres- } \\
\text { sion Scale to } \\
\text { measure depres- } \\
\text { sion level }\end{array}$ & $\begin{array}{l}\text { Recall: } 0.697 \text {; } \\
\text { precision: } \\
0.604 ; \mathrm{F} 1: \\
0.647\end{array}$ \\
\hline Weibo & Lin et al [33] & Stress & $\begin{array}{l}1 \text { billion Weibo } \\
\text { posts }\end{array}$ & $\begin{array}{l}\text { SVM, softmax } \\
\text { regression, gra- } \\
\text { dient-boosted } \\
\text { decision tree, } \\
\text { LASSO-MTL }{ }^{\mathrm{i}} \text {, } \\
\text { L2-MTL }^{\mathrm{j}}, \mathrm{cA}^{-} \\
\text {SO-MTL }^{\mathrm{k}}\end{array}$ & $\begin{array}{l}\text { CNN features } \\
\text { or word vector } \\
\text { representations } \\
\text { with hand-craft- } \\
\text { ed features }\end{array}$ & $\begin{array}{l}12 \text { stressor } \\
\text { events (eg, } \\
\text { marriage, fi- } \\
\text { nancial, ill- } \\
\text { ness, } \\
\text { school), } 6 \\
\text { stressor sub- } \\
\text { jects }\end{array}$ & $\begin{array}{l}30 \text { volunteers } \\
\text { manually anno- } \\
\text { tated the stres- } \\
\text { sor events and } \\
\text { subjects }\end{array}$ & $\begin{array}{l}10 \text {-fold cross } \\
\text { validation } \\
(\mathrm{F} 1>0.80)\end{array}$ \\
\hline Weibo & $\begin{array}{l}\text { Cheng et al } \\
{[34]}\end{array}$ & $\begin{array}{l}\text { Suicide risk, } \\
\text { depression, } \\
\text { anxiety, } \\
\text { stress }\end{array}$ & $\begin{array}{l}974 \text { partici- } \\
\text { pants' Weibo } \\
\text { posts, suicide } \\
\text { probability, } \\
\text { Weibo suicide } \\
\text { communication } \\
\text { (WSC), depres- } \\
\text { sion, anxiety, } \\
\text { and stress. }\end{array}$ & SVM & $\begin{array}{l}\text { Simplified Chi- } \\
\text { nese-LIWC }\end{array}$ & $\begin{array}{l}\text { Suicide risk, } \\
\text { emotional } \\
\text { distress (de- } \\
\text { pression, } \\
\text { anxiety, } \\
\text { stress), } \\
\text { WSC }\end{array}$ & $\begin{array}{l}\text { Survey and psy- } \\
\text { chological test } \\
\text { tools (ie, Sui- } \\
\text { cide Probability } \\
\text { Scale, Depres- } \\
\text { sion Anxiety } \\
\text { Stress Scales- } \\
21)\end{array}$ & $\begin{array}{l}\text { leave-one-out } \\
\text { cross-valida- } \\
\text { tion: suicide } \\
\text { probability } \\
\text { (AUC }=0.61, \\
P=.04) \text {, severe } \\
\text { anxiety } \\
(\text { AUC }=0.75, \\
P<.001)\end{array}$ \\
\hline
\end{tabular}




\begin{tabular}{|c|c|c|c|c|c|c|c|c|}
\hline \multirow[t]{2}{*}{ Data Source } & \multirow[t]{2}{*}{ Reference } & \multirow{2}{*}{$\begin{array}{l}\text { Mental } \\
\text { Health }\end{array}$} & \multirow{2}{*}{$\begin{array}{l}\text { Data Descrip- } \\
\text { tion }\end{array}$} & \multicolumn{5}{|l|}{ Machine learning } \\
\hline & & & & Model & Feature & Output & Annotation & Results \\
\hline Reddit & $\begin{array}{l}\text { Gkotsis et al } \\
{[35]}\end{array}$ & $\begin{array}{l}\text { Bipolar, } \\
\text { schizophre- } \\
\text { nia, anxiety, } \\
\text { depression, } \\
\text { self-harm, } \\
\text { suicide } \\
\text { watch, addic- } \\
\text { tion, crip- } \\
\text { pling alco- } \\
\text { holism, opi- } \\
\text { ates, autism }\end{array}$ & $1,014,660$ posts & $\begin{array}{l}\mathrm{CNN}, \mathrm{FF}^{1} \text {, lin- } \\
\text { ear regression, } \\
\mathrm{SVM}\end{array}$ & $\begin{array}{l}\text { Word vector } \\
\text { representation } \\
(16 \text { vector size })\end{array}$ & $\begin{array}{l}\text { Mental } \\
\text { health }\end{array}$ & $\mathrm{N} / \mathrm{A}^{\mathrm{m}}$ & $\begin{array}{l}\text { Accuracy: } \\
91.8 \% \text { (binary } \\
\text { classification } \\
\text { task), } 79.8 \% \\
\text { (multiclass clas- } \\
\text { sification task) }\end{array}$ \\
\hline $\begin{array}{l}\text { Facebook, } \\
\text { Twitter, In- } \\
\text { stagram, } \\
\text { Reddit }\end{array}$ & $\begin{array}{l}\text { Coppersmith } \\
\text { et al [36] }\end{array}$ & Suicide risk & $\begin{array}{l}197,615 \text { posts } \\
\text { from } 418 \text { users }\end{array}$ & $\begin{array}{l}\text { LSTM with at- } \\
\text { tention }\end{array}$ & $\begin{array}{l}\text { One-hot-vector } \\
\text { mapped to pre- } \\
\text { trained GloVe }\end{array}$ & Suicide risk & $\begin{array}{l}\text { Examining pub- } \\
\text { lic self-stated } \\
\text { data and using } \\
\text { data donated } \\
\text { through Our- } \\
\text { DataHelps.org }\end{array}$ & $\begin{array}{l}10 \text {-fold cross } \\
\text { validation } \\
(\mathrm{AUC}=0.94)\end{array}$ \\
\hline $\begin{array}{l}\text { Online Com- } \\
\text { munity - } \\
\text { Live Journal }\end{array}$ & $\begin{array}{l}\text { Saha et al } \\
{[37]}\end{array}$ & $\begin{array}{l}\text { Mental } \\
\text { health }\end{array}$ & $\begin{array}{l}620,060 \text { posts } \\
\text { from } 78,647 \\
\text { users }\end{array}$ & MTL & $\begin{array}{l}\text { Linguistic fea- } \\
\text { tures of LIWC; } \\
\text { topics by LDA }\end{array}$ & $\begin{array}{l}\text { Mental } \\
\text { health sub- } \\
\text { reddit (eg, } \\
\text { Abuse, } \\
\text { Anorexia, } \\
\text { Anxiety, } \\
\text { Bipolar disor- } \\
\text { der, Cutting, } \\
\text { Death, } \\
\text { Drugs, Eat- } \\
\text { ing disor- } \\
\text { ders, Insom- } \\
\text { nia, Pain, } \\
\text { Self-injury, } \\
\text { and Suicide) }\end{array}$ & N/A & $\begin{array}{l}\text { AUC }=0.94 \text { with } \\
\text { the community } \\
\text { on eating disor- } \\
\text { ders }\end{array}$ \\
\hline
\end{tabular}

${ }^{\mathrm{a}} \mathrm{AUC}$ : area under the curve.

${ }^{\mathrm{b}} \mathrm{CNN}$ : convolutional neural network.

${ }^{\mathrm{c}} \mathrm{SVM}$ : support vector machine.

${ }^{\mathrm{d}}$ Bi-LSTM: bidirectional long short-term memory.

e LASSO: least absolute shrinkage and selection operator.

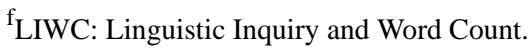

gDTSVM: decision tree support vector machine.

${ }^{\mathrm{h}_{\text {SNMDD: }}}$ social network mental disorder detection.

${ }^{\mathrm{i}}$ MTL: multitask learning.

$\mathrm{j}_{12-\mathrm{MTL}}$ : multitask learning considering 12 loss

k ASO-MTL: clustered alternating structure optimization multitask learning.

${ }^{1} \mathrm{FF}$ : feed-forward.

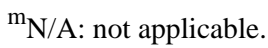

${ }^{\mathrm{n}} \mathrm{LDA}$ : latent Dirichlet allocation.

In the case of Twitter, Budenz et al [28] analyzed 1,270,902 tweets by searching bipolar and mental health terms. Before using a logistic regression analysis to classify whether a specific tweet was asking for any help or included terms associated with mental illness stigma, they repeatedly performed a series of sentiment analyses on 2047 randomly sampled tweets. The results obtained through 10 -fold cross-validation procedures showed an average area under the curve (AUC) of 0.83 when the term-frequency inverses document frequency weighted vector was employed as the input source.
Twitter tweets were also used to predict an association with psychological stressors, as one of the major causes of suicide. To prevent suicidal behaviors [29], the authors retrieved $1,962,766$ tweets based on 21 suicide-related keywords, manually annotated labels of a subset of 3263 tweets, and labeled the other 3000 tweets based on ML classifiers. A Twitter corpus-pretrained GloVe vector was employed to convert each token into a vector as an input of the convolutional neural network (CNN). The CNN model achieved an F1-score of 83\%, which outperformed SVM, extra trees, and other ML algorithms. 
In addition, stress level, as one of the pervasive causes of mental health conditions, was predicted on social media platforms, including Twitter and Facebook [30]. Linguistic characteristics were extracted from a total of 601 users' social media posts using the Linguistic Inquiry and Word Count (LIWC) tool, latent Dirichlet allocation (LDA), and stress lexicon. To predict stress, the authors applied linear regression with regularization methods, and validated the model performance based on sociodemographic variables (eg age, gender, race, income, and education) and social media language using the Pearson correlation coefficient $(r)$. The content analysis indicated significant differences in language expressions among social media platforms.

Shuai et al [31] collected posts from both Facebook and Instagram to detect social network mental disorders (SNMD), which include several side effects such as cyber-relationship addiction or net compulsion. They developed and employed SNMD questionnaire items to classify each participant into specific types of SNMD. Among more than 3100 participants, 389 respondents were regarded as having SNMD. Social interaction, personal profile, and duration extracted by each participant's social activity logs were employed as input features of ML models. The proposed model, which was organized by new tensor techniques and latent features, achieved more than $83 \%$ accuracy in identifying whether a specific user has SNMD.

One of the notable approaches in this area is a visual-oriented approach. Reece and Danforth [32] employed 43,950 images from 166 Instagram users to detect posts related to depression. Based on the results of the Center for Epidemiologic Studies Depression Scale questionnaire (CES-D), a total of 71 users revealed that they experienced depression. Moreover, both Instagram usernames and history were collected from crowd workers who responded to the CES-D. They extracted metadata (eg, the number of comments, "likes"), color properties (eg, hue, saturation, value), and the total number of faces from the collected photographs to investigate whether users suffer from depression.

Based on the guidelines of De Choudhury et al [38], Reece and Danforth decided to integrate the users' recent posts presented on a specific (single) day rather than using their entire posts. Through a random forest classifier, they achieved a relatively high recall score of identifying the target class at $70 \%$ in 100 observations. The results indicated that the photos posted by depressed users were more likely to be bluer, grayer, and darker, and to receive fewer likes. However, as a limitation of the study, they pointed out that depression is a form of general clinical status, indicating a need for fine-tuning the questionnaires for specific diagnosis.

Lin et al [33] collected approximately 1 billion tweets from the Chinese social media platform Weibo, and proposed ML multitask models to detect both stressor events and six subjects. The event was categorized into 12 different labels, including marriage, financial, illness, and school. Each tweet was first labeled as stress-related. The tweets were categorized into one of the stressor events and subject categories by 30 volunteers. The performance of classifying a stressor event or subject was represented with various classifiers such as SVM, softmax regression, and gradient decision. The model performance was not clearly presented; however, it was stated that the F1-score reached over $80 \%$ in the event detection task.

A mental illness is often accompanied by another mental illness as a so-called "comorbidity," which refers to the simultaneous presence of one or more mental or physical disorders. From this viewpoint, Saha et al [37] developed a joint learning model, which was generated by multitask learning to simultaneously identify co-occurring social media communities related to mental health with consideration of the correlation between the communities. Based on 620,060 posts of 78,647 users in 247 online communities, 12 major mental health-related topics were employed in the categorization standards from "Live Journal" (eg, Abuse, Anorexia, Anxiety, Bipolar disorder, etc). Using these data, two features were extracted as inputs: language style (from the LIWC) and topics (from the results of LDA). In general, the proposed model outperformed single-task learning [39] and multitask learning [40] for 9 out of 12 and 8 out of 12 categories, respectively. Moreover, the model achieved an AUC of 0.94 with the community on eating disorders.

Cheng et al [34] utilized Weibo data to assess the levels of suicide risk and emotional distress such as depression, anxiety, and stress. For this purpose, the researchers completed an internet survey and gathered 974 respondents' Weibo posts, the scores of mental health (ie, suicide probability, depression, anxiety, and stress) through psychological investigation tools, and Weibo Suicide Communication (WSC), which examined whether respondents had told others that they wanted to commit suicide through Weibo over the past 12 months. SVM was applied for a binary classification of five suicide risk factors (suicide probability, depression, anxiety, stress, and WSC), including 72 linguistic features of Simplified Chinese-LIWC from the respondents' Weibo posts as independent variables. The model efficiently classified the respondents having a high suicide probability (AUC=0.61, $P=.04$ ) and severe anxiety (AUC $=0.75, P<.001$ ) among those who had WSC with leave-one-out cross-validation procedures.

Gkotsis et al [35] collected user-oriented data from the Reddit community to develop deep-learning models for classifying posts according to mental disorder topics. After an expert panel made a decision on whether a specific post contains mental health-related issues, they collected $10,146,60$ posts and extracted 11 mental disorder themes, including a nonmental health conditions. To classify whether a specific post belongs to one of the mental health topics, they employed a CNN model with two parallel classification approaches: binary and multiclass classifications. The word vectors of each token extracted from post texts were used as input. The results of the model demonstrated that the CNN classifier showed $91.8 \%$ and $79.8 \%$ accuracy in binary and multiclass classification tasks, respectively.

Early estimation of a person's suicide risk is also an important issue in our society. In accordance with this point, Coppersmith et al [36] employed social media data to predict the level of suicide risk using a long short-term memory (LSTM) model. Two different datasets were collected: one from donated data through OurDataHelps.org, which included social media data 
of suicide victims (eg, Facebook, Instagram, Twitter), and the other from Harman Dredze [41], which provided Twitter data from users who mentioned their past suicide attempts in tweets. A total of 197,615 posts from 418 users were obtained. Based on the pretrained GloVe embeddings to feed sequences of word vectors into the bidirectional LSTM model, an AUC of 0.94 was achieved through 10-fold cross-validation procedures.

\section{Discussion}

This study involved a bibliometric analysis on the publications related to ML and mental health in social media from 2015 to 2020 with two citation databases, WoS and Scopus, as well as a trend review analysis. Although several prior studies have investigated mental illness, the majority of these studies employed both clinical and physical health care approaches. Along with these studies, social media is considered as one of the most important spaces for effectively and efficiently addressing individuals' mental health issues [42]. Furthermore, with rapidly improved ML and big data techniques, both the significance and importance of employing social media and online communications are being consistently emphasized.

Rapidly and consistently increasing publication and citation numbers indicate that there is growing attention and interest in this research area. Among several publication venues, Lecture Notes in Computer Science (Scopus) and Journal of Medical Internet Research (WoS) were the most productive publication sources in this field. Moreover, Harvard University and Georgia Institute of Technology in the United States, and Tsinghua University and the Chinese Academy of Sciences in China were listed as the most vigorous institutes. For individual researchers, Professor Munmun De Choudhury from Georgia Institute of Technology emerged as the most productive and well-known researcher in this field, with 15 publications to her credit. Regarding publications, using social media data in predicting depression was Prof De Choudhury's first step in this area [38], which allowed her affiliated nation (United States) to be the most productive country in this field. Computer science, medicine, and medical informatics were identified as the core research subjects, along with several other related subjects such as psychology, social science, and neuroscience. This suggests that this research area tends to require integrated or multidisciplinary approaches for gaining a better understanding of each research topic. In addition, the keyword co-occurrence network graph highlighted the representative ML techniques and social media for this multidisciplinary area.

Subsequently, we conducted a trend analysis review on highly cited articles, and notable research trends were identified. The highly cited articles tended to employ user-generated content in diverse forms, including text, images, and other metadata, for specific mental disorders. Because no ground truth labels exist for users who have mental disorders, the majority of studies adopted a crowdsourcing survey with a medical-oriented approach and consideration of the participants' agreements in using their social media accounts [30-32,34]. Moreover, several scholars have employed user-oriented features, including users' demographic profiles and activity logs, in social media (eg, comments, likes) to arrive at both academic and practical contributions [30,31].

Based on the employed approaches with several highly cited articles, three main implications for discussion can be derived. First, the majority of the articles stated privacy and ethical issues as key considerations in using ML for specific mental illness in social media $[23,30,32,36]$. Although they met both research ethical guidelines and participants' agreements in using their social media data, there were notable adverse reactions from several participants in sharing their social media information [32]. Moreover, compared to other issues in this area, both privacy and ethical issues are considered to be real issues requiring more academic and practical work [23]. Thus, researchers must make efforts to effectively consider these threats, which can negatively affect data providers and leave room for abusing ML techniques. Second, because there is potential for misclassified ground truth data, there should be more detailed and systematic examinations in building the early stage of datasets [28,29,34,36]. Third, because users' expressions on social media can consistently change over time, time-oriented approaches with differing perspectives toward users' activities and expressions should be considered in providing a better understanding in this area [32].

Considering these discussion points, a few limitations of this study remain. Although we employed WoS and Scopus as our subjects, which are both widely used academic databases globally, there can be other medical-oriented databases that may provide more significant academic and practical information. Moreover, considering more recent and applicable statistical or natural language processing techniques (such as exploratory factor analysis [23] or topic modeling [20,23]), future research should aim at obtaining deeper and comprehensive knowledge with more creative and significant approaches through various data sources of each article.

With the increase of AI applications in real-word health care settings [1], there have been numerous attempts to overcome the limitations of offline consultations such as wearable fitness trackers [43], mobile health apps [44], and conversational agents for patients' mental health and wellness [1,45]. Moreover, since the use of social media has been widely adopted in health care [46], we believe that our analysis may trigger all stakeholders to further consider how to employ ML approaches toward mental health in social media. In addition, when applying social media data to clinical settings, there is a need to address different characteristics of social media platforms by utilizing the substantial research background.

\section{Acknowledgments}

This work was supported by an Institute of Information and Communications Technology Planning \& Evaluation (IITP) grant funded by the Korean government (Ministry of Science and ICT; No. 2020-0-00990, Platform Development and Proof of High Trust \& Low Latency Processing for Heterogeneous Atypical Large Scaled Data in 5G-IoT Environment). This research was also supported by the National Research Foundation of Korea funded by the Korean Government (NRF-2020R1C1C1004324). 


\section{Conflicts of Interest}

None declared.

\section{Multimedia Appendix 1}

Complete publication lists.

[XLSX File (Microsoft Excel File), 64 KB-Multimedia Appendix 1]

\section{References}

1. Davenport T, Kalakota R. The potential for artificial intelligence in healthcare. Future Healthc J 2019 Jun;6(2):94-98 [RREE Full text] [doi: 10.7861/futurehosp.6-2-94] [Medline: 31363513]

2. Kahan J. Mobilizing AI for health to fight against COVID-19. Microsoft blogs. 2020 Apr 09. URL: https://blogs. microsoft.com/on-the-issues/2020/04/09/ai-for-health-covid-19 [accessed 2020-09-18]

3. Castelo M. The Future of Artificial Intelligence in Healthcare. HealthTech. 2020 Feb 26. URL: https://healthtechmagazine. net/article/2020/02/future-artificial-intelligence-healthcare [accessed 2020-09-18]

4. Hao K. Doctors are using AI to triage covid-19 patients. The tools may be here to stay. MIT Technology Review. 2020 Apr 23. URL: https://www.technologyreview.com/2020/04/23/1000410/ai-triage-covid-19-patients-health-care/ [accessed 2020-09-18]

5. Yu K, Beam AL, Kohane IS. Artificial intelligence in healthcare. Nat Biomed Eng 2018 Oct;2(10):719-731. [doi: 10.1038/s41551-018-0305-z] [Medline: 31015651]

6. Shahid N, Rappon T, Berta W. Applications of artificial neural networks in health care organizational decision-making: A scoping review. PLoS One 2019;14(2):e0212356 [FREE Full text] [doi: 10.1371/journal.pone.0212356] [Medline: 30779785]

7. World Mental Health Day: an opportunity to kick-start a massive scale-up in investment in mental health. World Health Organization. 2020 Aug 27. URL: https://www.who.int/news-room/detail/

27-08-2020-world-mental-health-day-an-opportunity-to-kick-start-a-massive-scale-up-in-investment-in-mental-health [accessed 2020-09-18]

8. GBD 2017 Disease Injury Incidence Prevalence Collaborators. Global, regional, and national incidence, prevalence, and years lived with disability for 354 diseases and injuries for 195 countries and territories, 1990-2017: a systematic analysis for the Global Burden of Disease Study 2017. Lancet 2018 Nov 10;392(10159):1789-1858 [FREE Full text] [doi: 10.1016/S0140-6736(18)32279-7] [Medline: 30496104]

9. Batterham PJ, Calear AL. Preferences for internet-based mental health interventions in an adult online sample: findings from an online community survey. JMIR Ment Health 2017 Jun 30;4(2):e26 [FREE Full text] [doi: 10.2196/mental.7722] [Medline: 28666976]

10. Brunette M, Achtyes E, Pratt S, Stilwell K, Opperman M, Guarino S, et al. Use of smartphones, computers and social media among people with SMI: opportunity for intervention. Community Ment Health J 2019 Aug 8;55(6):973-978. [doi: 10.1007/s10597-019-00431-7] [Medline: 31175518]

11. Naslund JA, Bondre A, Torous J, Aschbrenner KA. Social media and mental health: benefits, risks, and opportunities for research and practice. J Technol Behav Sci 2020 Sep;5(3):245-257. [doi: 10.1007/s41347-020-00134-x] [Medline: 33415185]

12. Breland JY, Quintiliani LM, Schneider KL, May CN, Pagoto S. Social media as a tool to increase the impact of public health research. Am J Public Health 2017 Dec;107(12):1890-1891. [doi: 10.2105/AJPH.2017.304098] [Medline: 29116846]

13. Higgins JPT, Altman DG. Assessing risk of bias in included studies. In: Higgins JPT, Green S, editors. Cochrane Handbook for Systematic Reviews of Interventions: Cochrane Book Series. Chichester, UK: John Wiley \& Sons Ltd and The Cochrane Collaboration; 2008:187-241.

14. Seabrook EM, Kern ML, Rickard NS. Social networking sites, depression, and anxiety: a systematic review. JMIR Ment Health 2016 Nov 23;3(4):e50 [FREE Full text] [doi: 10.2196/mental.5842] [Medline: 27881357]

15. Pham MT, Rajić A, Greig JD, Sargeant JM, Papadopoulos A, McEwen SA. A scoping review of scoping reviews: advancing the approach and enhancing the consistency. Res Synth Methods 2014 Dec;5(4):371-385 [FREE Full text] [doi: 10.1002/jrsm.1123] [Medline: 26052958]

16. Shatte ABR, Hutchinson DM, Teague SJ. Machine learning in mental health: a scoping review of methods and applications. Psychol Med 2019 Jul;49(9):1426-1448. [doi: 10.1017/S0033291719000151] [Medline: 30744717]

17. Chancellor S, Baumer EPS, De Choudhury M. Who is the human in human-centered machine learning? The case of predicting mental health from social media. 2019 Nov 07 Presented at: ACM on Human-Computer Interaction, 3 (CSCW); November 2019; Texas, USA p. 1-32. [doi: 10.1145/3359249]

18. Chancellor S, De Choudhury M. Methods in predictive techniques for mental health status on social media: a critical review. NPJ Digit Med 2020;3:43. [doi: 10.1038/s41746-020-0233-7] [Medline: 32219184]

19. Pritchard A. Statistical bibliography or bibliometrics. J Document 1969;25(4):348-349.

20. Chen X, Wang S, Tang Y, Hao T. A bibliometric analysis of event detection in social media. Online Inf Rev 2019 Feb 11;43(1):29-52. [doi: 10.1108/oir-03-2018-0068] 
21. Zyoud SH, Sweileh WM, Awang R, Al-Jabi SW. Global trends in research related to social media in psychology: mapping and bibliometric analysis. Int J Ment Health Syst 2018;12:4 [FREE Full text] [doi: 10.1186/s13033-018-0182-6] [Medline: 29387147]

22. dos Santos BS, Steiner MTA, Fenerich AT, Lima RHP. Data mining and machine learning techniques applied to public health problems: A bibliometric analysis from 2009 to 2018. Comput Indust Eng 2019 Dec;138:106120. [doi: 10.1016/j.cie.2019.106120]

23. Tran BX, McIntyre RS, Latkin CA, Phan HT, Vu GT, Nguyen HLT, et al. The current research landscape on the artificial intelligence application in the management of depressive disorders: a bibliometric analysis. Int J Environ Res Public Health 2019 Jun 18;16(12):2150 [FREE Full text] [doi: 10.3390/ijerph16122150] [Medline: 31216619]

24. Wang J, Deng H, Liu B, Hu A, Liang J, Fan L, et al. Systematic evaluation of research progress on natural language processing in medicine over the past 20 years: bibliometric study on PubMed. J Med Internet Res 2020 Jan 23;22(1):e16816 [FREE Full text] [doi: 10.2196/16816] [Medline: $\underline{32012074]}$

25. Kim J, Lee J, Park E, Han J. A deep learning model for detecting mental illness from user content on social media. Sci Rep 2020 Jul 16;10(1):11846. [doi: 10.1038/s41598-020-68764-y] [Medline: 32678250]

26. Lee $\mathrm{P}, \mathrm{Su} \mathrm{H}$. Investigating the structure of regional innovation system research through keyword co-occurrence and social network analysis. Innovation 2014 Dec 17;12(1):26-40. [doi: 10.5172/impp.12.1.26]

27. Bardus M, El Rassi R, Chahrour M, Akl EW, Raslan AS, Meho LI, et al. The use of social media to increase the impact of health research: systematic review. J Med Internet Res 2020 Jul 06;22(7):e15607 [FREE Full text] [doi: 10.2196/15607] [Medline: 32628113]

28. Budenz A, Klassen A, Purtle J, Yom Tov E, Yudell M, Massey P. Mental illness and bipolar disorder on Twitter: implications for stigma and social support. J Ment Health 2020 Apr;29(2):191-199. [doi: 10.1080/09638237.2019.1677878] [Medline: 31694433]

29. Du J, Zhang Y, Luo J, Jia Y, Wei Q, Tao C, et al. Extracting psychiatric stressors for suicide from social media using deep learning. BMC Med Inform Decis Mak 2018 Jul 23;18(Suppl 2):43 [FREE Full text] [doi: 10.1186/s12911-018-0632-8] [Medline: 30066665]

30. Guntuku S, Buffone A, Jaidka K, Eichstaedt J, Ungar L. Understanding and measuring psychological stress using social media. 2019 Jul Presented at: Thirteenth International AAAI Conference on Web and Social Media; July 2019; Munich, Germany p. 214-225.

31. Shuai H, Shen C, Yang D, Lan Y, Lee W, Yu P, et al. A comprehensive study on social network mental disorders detection via online social media mining. IEEE Trans Knowl Data Eng 2018 Jul 1;30(7):1212-1225. [doi: 10.1109/TKDE.2017.2786695]

32. Reece AG, Danforth CM. Erratum to: Instagram photos reveal predictive markers of depression. EPJ Data Sci 2017 Sep 5;6(1):1-12. [doi: 10.1140/epjds/s13688-017-0118-4]

33. Lin H, Jia J, Nie L, Shen G, Chua T. What does social media say about your stress? 2016 Jul Presented at: Twenty-Fifth International Joint Conference on Artificial Intelligence (IJCAI'16); July 2016; New York, USA p. 3775-3781 URL: https:/ /dl.acm.org/doi/abs/10.5555/3061053.3061147

34. Cheng Q, Li TM, Kwok C, Zhu T, Yip PS. Assessing suicide risk and emotional distress in Chinese social media: a text mining and machine learning study. J Med Internet Res 2017 Jul 10;19(7):e243 [FREE Full text] [doi: 10.2196/jmir.7276] [Medline: 28694239]

35. Gkotsis G, Oellrich A, Velupillai S, Liakata M, Hubbard TJP, Dobson RJB, et al. Characterisation of mental health conditions in social media using Informed Deep Learning. Sci Rep 2017 Mar 22;7:45141. [doi: 10.1038/srep45141] [Medline: 28327593]

36. Coppersmith G, Leary R, Crutchley P, Fine A. Natural language processing of social media as screening for suicide risk. Biomed Inform Insights 2018;10:1178222618792860 [FREE Full text] [doi: 10.1177/1178222618792860] [Medline: 30158822]

37. Saha B, Nguyen T, Phung D, Venkatesh S. A Framework for Classifying Online Mental Health-Related Communities With an Interest in Depression. IEEE J Biomed Health Inform 2016 Jul;20(4):1008-1015. [doi: 10.1109/JBHI.2016.2543741] [Medline: 27008680]

38. De Choudhury M, Counts S, Horvitz E. Predicting postpartum changes in emotion and behavior via social media. 2013 Apr Presented at: SIGCHI conference on human factors in computing systems; April 2013; Paris, France p. $3267-3276$. [doi: $10.1145 / 2470654.2466447]$

39. Liu J, Chen J, Ye J. Large-scale sparse logistic regression. 2009 Jun Presented at: 15th ACM SIGKDD international conference on knowledge discovery and data mining; June 2009; Paris, France p. 547-556 URL: https://dl.acm.org/doi/abs/ $\underline{10.1145 / 1557019.1557082}$

40. Liu J, Ji S, Ye J. Multi-task feature learning via efficient '2, 1-norm minimization. 2009 Jun Presented at: Twenty-Fifth Conference on Uncertainty in Artificial Intelligence; June 2009; Quebec, Canada p. 339-348 URL: https://dl.acm.org/doi/ $\underline{10.5555 / 1795114.1795154}$

41. Harman G, Dredze M. Measuring post traumatic stress disorder in Twitter. 2014 May Presented at: Eighth International AAAI Conference on Weblogs and Social Media; May 2014; Michigan, USA. 
42. De Choudhury M. Role of social media in tackling challenges in mental health. 2013 Oct Presented at: 2nd international workshop on socially-aware multimedia; October 2013; Barcelona, Spain p. 49-52. [doi: 10.1145/2509916.2509921]

43. Henriksen A, Haugen Mikalsen M, Woldaregay AZ, Muzny M, Hartvigsen G, Hopstock LA, et al. Using fitness trackers and smartwatches to measure physical activity in research: analysis of consumer wrist-worn wearables. J Med Internet Res 2018 Mar 22;20(3):e110 [FREE Full text] [doi: 10.2196/jmir.9157] [Medline: 29567635]

44. Grist R, Porter J, Stallard P. Mental health mobile apps for preadolescents and adolescents: a systematic review. J Med Internet Res 2017 May 25;19(5):e176 [FREE Full text] [doi: 10.2196/jmir.7332] [Medline: 28546138]

45. Provoost S, Lau HM, Ruwaard J, Riper H. Embodied conversational agents in clinical psychology: a scoping review. J Med Internet Res 2017 May 09;19(5):e151 [FREE Full text] [doi: 10.2196/jmir.6553] [Medline: 28487267]

46. Ukoha C, Stranieri A. Criteria to measure social media value in health care settings: narrative literature review. J Med Internet Res 2019 Dec 16;21(12):e14684 [FREE Full text] [doi: 10.2196/14684] [Medline: 31841114]

\author{
Abbreviations \\ AI: artificial intelligence \\ AUC: area under the curve \\ CES-D: Center for Epidemiologic Studies Depression Scale \\ CNN: convolutional neural network \\ HCML: human-centered machine learning \\ LDA: latent Dirichlet allocation \\ LIWC: Linguistic Inquiry and Word Count \\ LSTM: long short-term memory \\ ML: machine learning \\ SNMD: social network mental disorders \\ SVM: support vector machine \\ WoS: Web of Science \\ WSC: Weibo Suicide Communication
}

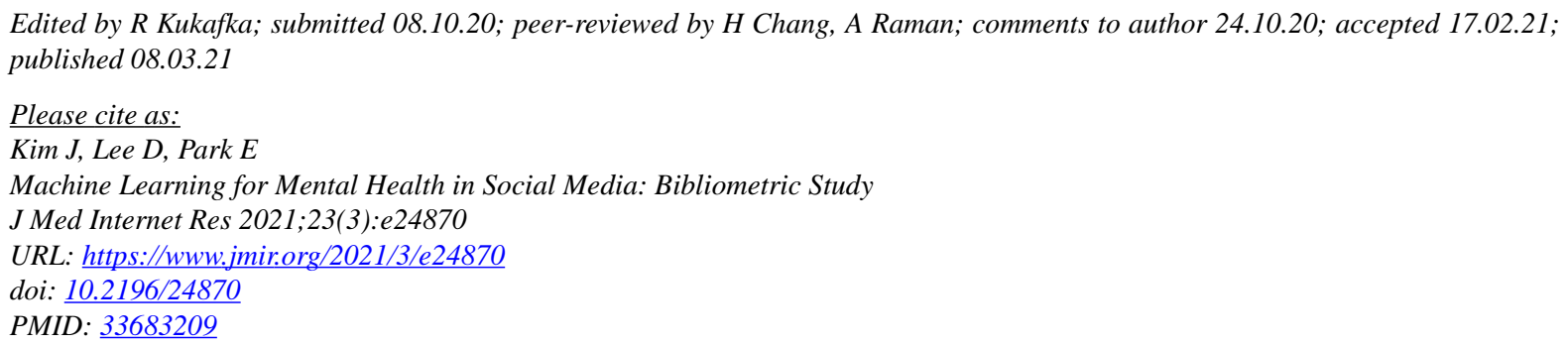

(CJina Kim, Daeun Lee, Eunil Park. Originally published in the Journal of Medical Internet Research (http://www.jmir.org), 08.03.2021. This is an open-access article distributed under the terms of the Creative Commons Attribution License (https://creativecommons.org/licenses/by/4.0/), which permits unrestricted use, distribution, and reproduction in any medium, provided the original work, first published in the Journal of Medical Internet Research, is properly cited. The complete bibliographic information, a link to the original publication on http://www.jmir.org/, as well as this copyright and license information must be included. 